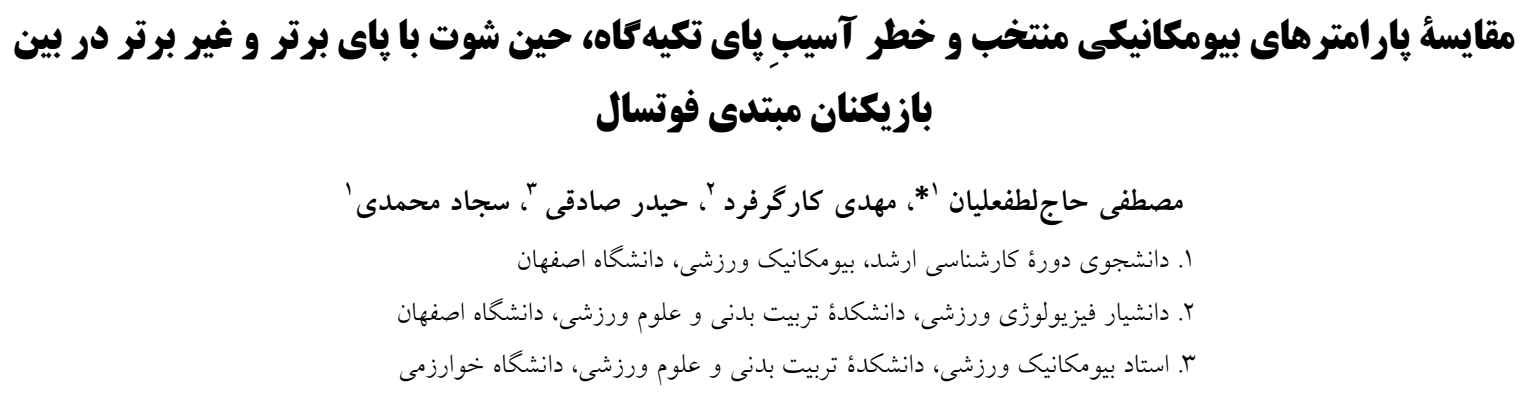

تاريخ يذيرش مقاله: IR IF/N/IF

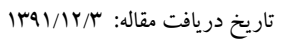

קكيله

با توجه به تأثير موقعيت قراركيرى ياى تكيه كاه بهمنزله عاملى مؤثر بر موفقيت شوت و بروز آسيب در مفصل مج با، هدف اين تحقيق، مقايسة منتخبى از

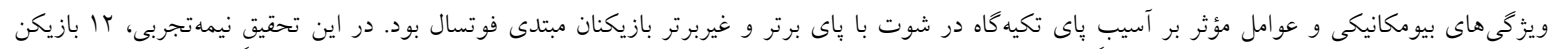

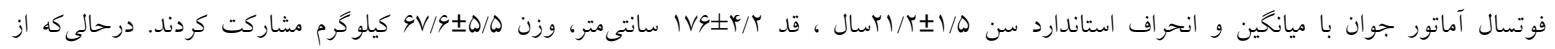

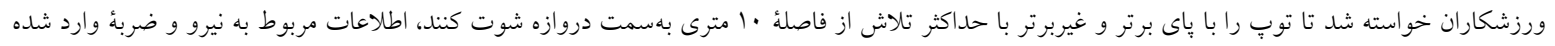

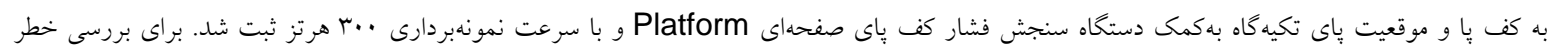

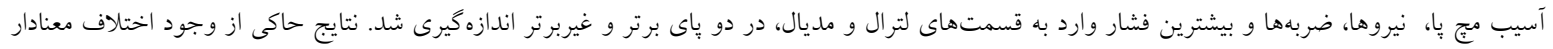

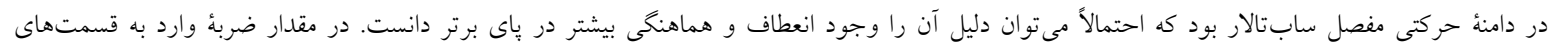

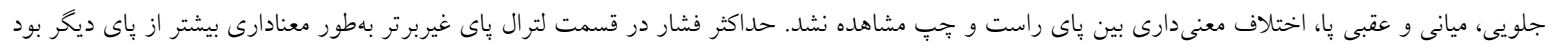

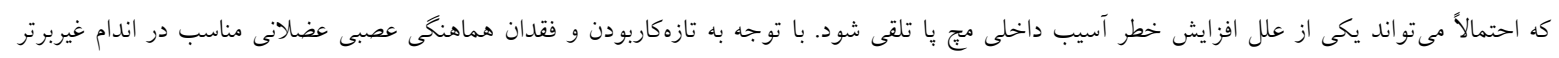

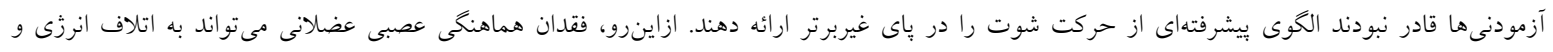

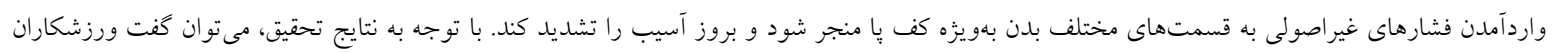

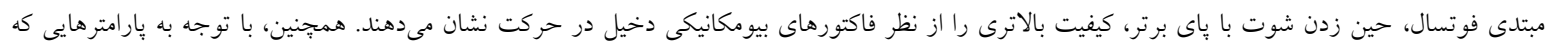

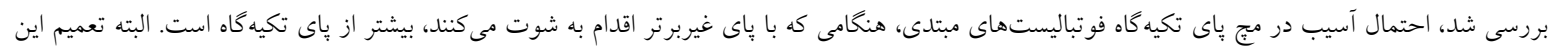

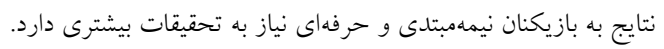

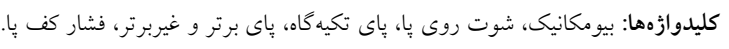

\title{
Comparison of selected biomechanical parameters and support foot injury during soccer instep kick by preferred and non-preferred lower limbs of the novice players
}

\section{Hajlotfalian, $\mathbf{M}^{1}$., Kargarfard, $\mathbf{M}^{2}$., Sadeghi, $\mathrm{H}^{3}$., Mohammadi, $\mathrm{S}^{1}$.}

1. M. Sc. Faculty of Physical Education and Sport Sciences, University of Isfahan

2. Associate Professor, Faculty of Physical Education and Sport Sciences, Isfahan University

3. Full Professor, Sports Biomechanic, Faculty of Physical Education and Sport Sciences, Kharazmi University, Iran

\begin{abstract}
Support foot position is one of the important factors which can affect instep kick quality and ankle joint injury. The aims of this study were to examine the support foot parameters in maximal instep kicking with the preferred and the non-preferred leg and research on risk factors for ankle joint injury in amateur futsal players. In this semi experimental study, twelve young and novice futsal players performed maximal speed place kicks with the preferred and the non-preferred leg. Average of the subjects individual characteristic have contained, age : $21 / 2 \pm 1 / 5$ years, weight : $67 / 6 \pm 5 / 5 \mathrm{Kg}$ and height : $176 \pm 4 / 2 \mathrm{~cm}$. Athletes were kicking the ball towards the goal from 10 meters. amount of support foot pressure were calculated at $300 \mathrm{~Hz}$. To calculate injury of ankle joint, Forces, impulses, position of the fixed leg and maximum pressure of the lateral and medial portions of the foots were determined. Results indicated a significant difference in amount of subtalar joint range of motion. Higher range of motion in dominant leg were caused by a greater flexibility and coordination in this side of the body. In addition there were no significant different in impact of anterior, middle and posterior portion of fixed
\end{abstract}

*. mostafa.h.lotfalian@gmail.com 
legs between two support foot. In contrast the maximum pressure in lateral side of non-preferred leg, was significantly more than other foot. In addition, the maximum force in non-preferred leg occurred prior then other foot. These problems can exacerbate ankle injury risk. Because the subjects were novice and they do not have appropriate neuromuscular coordination, can't show a good movement pattern in non preferred leg. Hence, the absence of neuromuscular coordination can lead to energy loss and high pressure coming into the body, especially the feet and it can exacerbate injuries. In a nutshell, amateur subjects indicated higher quality of biomechanical parameters in support foot of the preferred leg. Also likelihood of ankle sprain in dominant foot was less than other. Also generalization of these results to semi-professional and professional players, need further research.

Keywords: Biomechanics, Instep Kick, Support Foot, Preferred and Non-Preferred Foot, Foot Pressure.

مقدمه

ساختار متحرى مفصل مج ٍِّ بهدليل آنكه وزن كل بدن را تحمل مى كند، از آسيبِيذيرترين مفاصلٍ بدن حين انجام حركات ورزشى است (1). مستند شده است كه سالانه بيشاز دو بيليون دلار صرفِ درمان و بازتوانى مبتلايان به اين آسيب مىشود (Y). بروز اين آسيب در پياى تكيه كاه، مىتواند زمانى كه ورزشكار در حال انجام

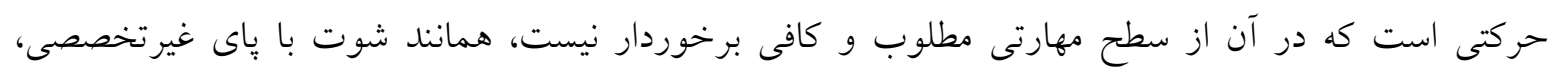

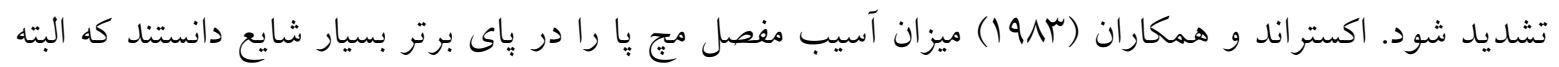

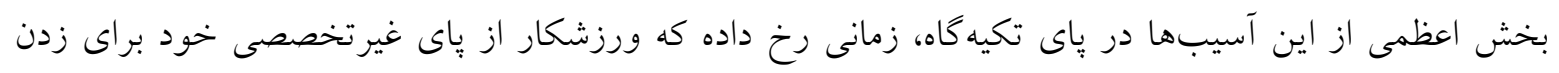
شوت استفاده كرده است. سن، جنس، مصدوميتهاى قبلى، قد، دامنهُ حركتى مفاصل و ميزان تعادل ايستا و يويا تهاي برخى از عواملى هستند كه بر بروز آسيب مجج پا تأثير مى گذارند و محققان بسيارى تأثير آنها را بررسى كردهاند

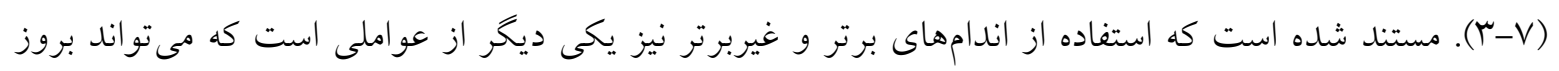
آسيب در مفصل مج را تحت تأثير قرار دهد. براى مثال، برخى محققان، درباب احتمال بروز آسيب در يّاى برتر و و

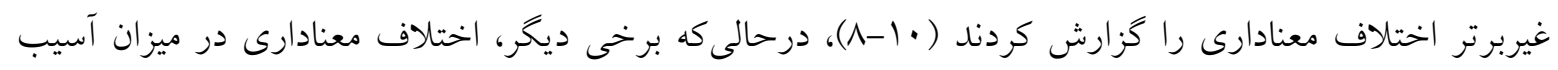

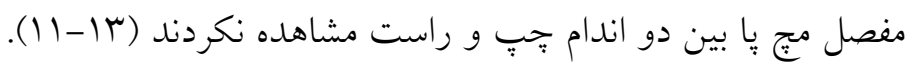

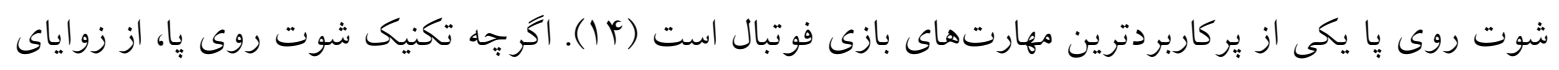

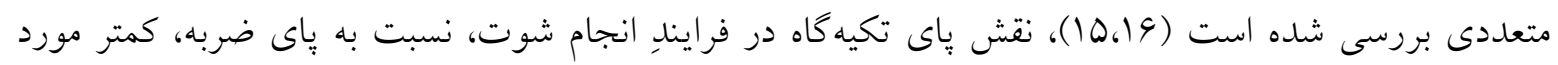
توجه بوده و بيشتر مطالعاتى كه در اين زمينه انجام شده، در زمينه محاسبهُ نيروى عكسالعمل زمين بوده است

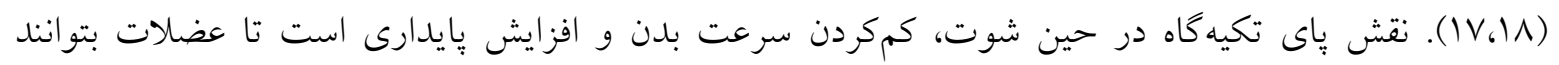
به خوبى نيرو توليد كنند (ع) (1). تعداد محدودى ورزشكار در سطح جهان وجود دارند كه مىتوانند توبٍ را با هردو يا، با يك كيفيت شوت كنند (19). به نظر مىرسد دليل اصلى ناتوانى ورزشكاران براى زدن شوتِّ باكيفيت، با پاى غيربرتر، شكل نخحفتن الخوى حركت در بدن ورزشكاران بهطور صحيح است. بنابراين، اين مسئله در

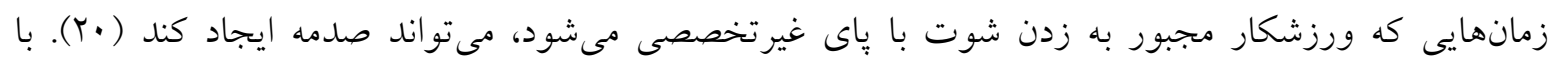
توجه به اينكه در حين بروز يِيجخوردكى مجج يا، توازن در فشار اعمالشده بر بخشهاى مختلف كف يا بههم

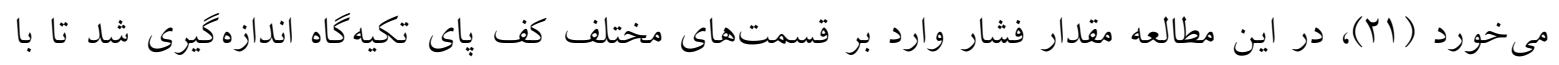


مقايسٔ بارامترهاى بيومكانيكى در باى برتر و غيربرتر، فرضئُ بالابودن احتمال بروز آسيب در بإى تكيهكاه حين

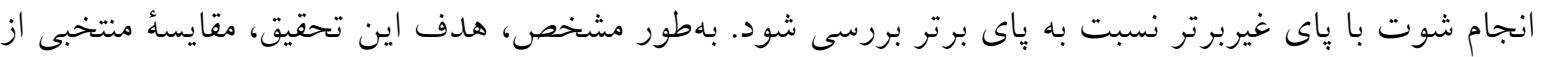

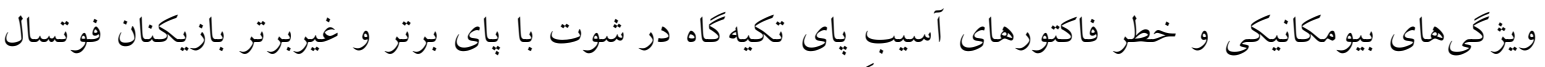

\section{روششناسى}

جامعة آمارى اين تحقيق نيمهتجربى را دانشجويان رشتئ تربيت بدنى و علوم ورزشى دانشكاه اصفهان تشكيل

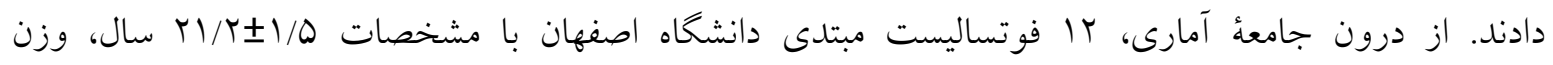
ند

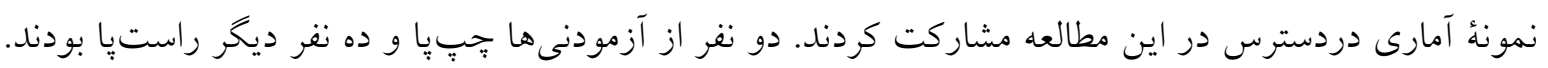

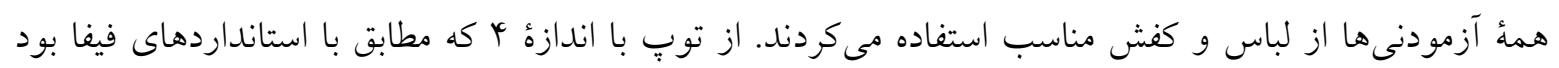

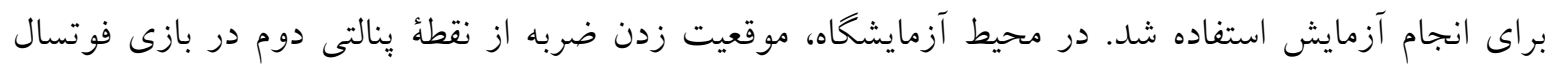

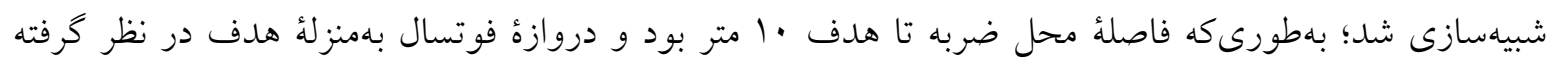

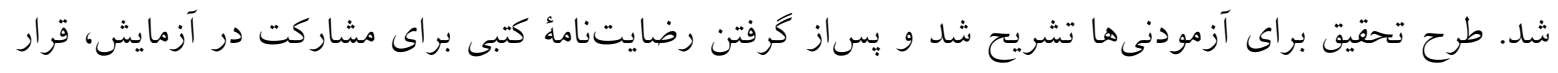

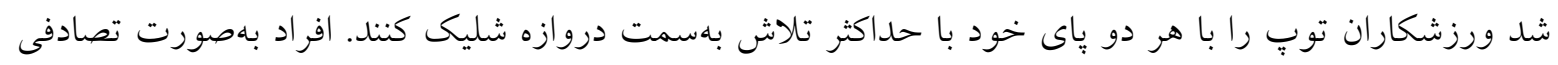

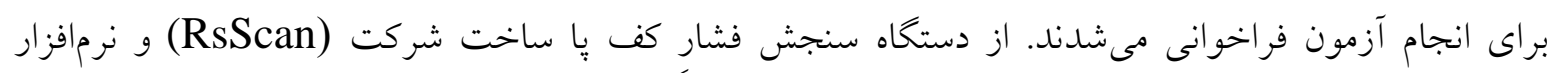

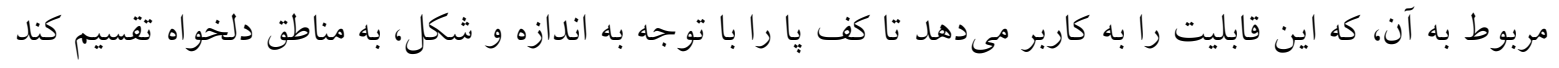

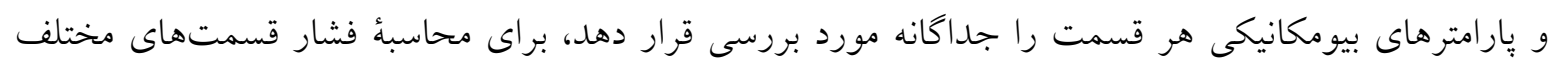

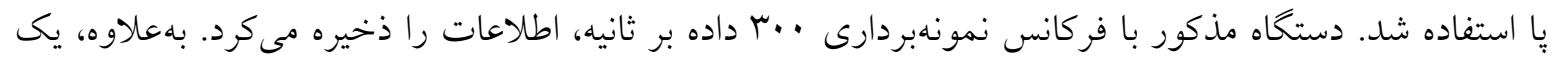

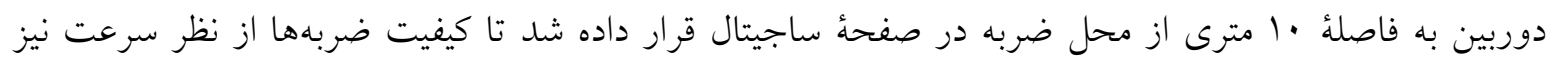

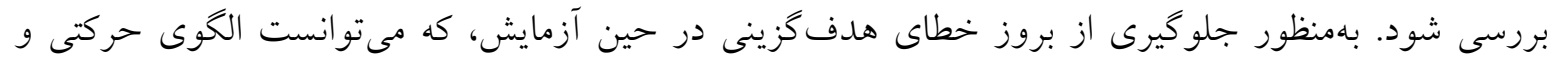

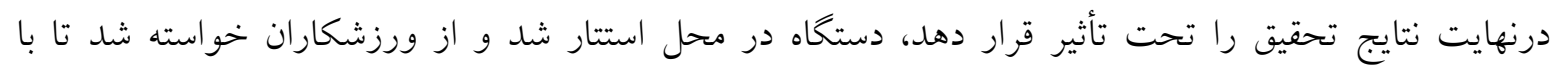

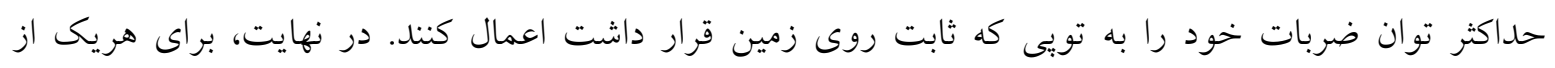

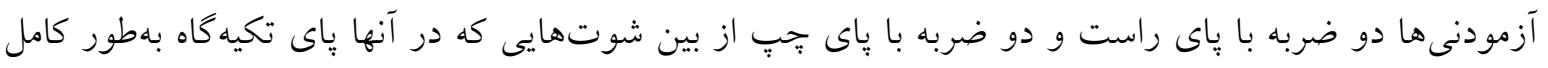

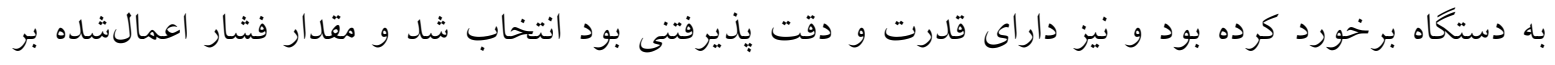

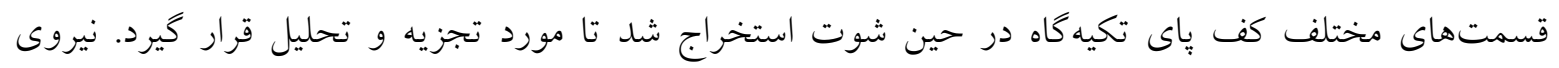

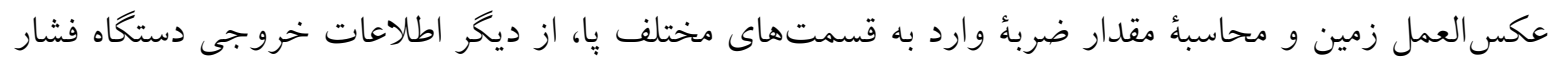


ֶِا' است كه از اطلاعات بهدستآمده از كف ياى تكيه كاه به كمك نرمافزار به سه قسمت عقبى، ميانى و جلويى تقسيم، و مقدار ضربهُ وارد به اين مناطق در بازهُ زمان محاسبه شد و در دو پِاى تكيه گاه برتر و غيربرتر در حين زدن شوت روى يا مقايسه شد. معادلات بهكار رفته براى محاسبة يار امترها به شرح ذيل است:

$$
\begin{aligned}
& \text { رابطة | }
\end{aligned}
$$

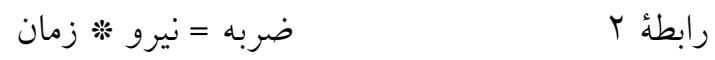

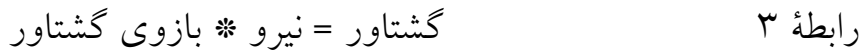

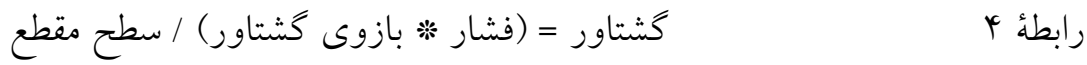

وضعيت توزيع دادهها با استفاده از آزمون كالموكروف - اسمرينوف بررسى شد. از آزمون t زوجى براى مقايسٔ آمارى متغيرها بين دو اندام برتر و غيربرتر در سطح معنى دارى PS

\section{يافتهها}

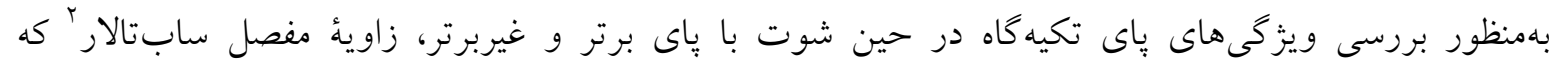

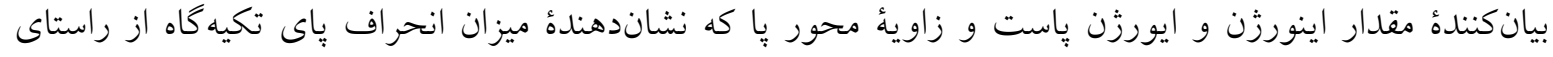
مستقيم است، در بين دو حركت مقايسه و اختلاف معنادار قابل توجهى در بين دو پا مشاهده شد. ضمن اينكه ضربهُ وارد به سه قسمت جلويى، ميانى و عقبى پا نيز تحت بررسى قرار گرفت و اختلاف معنادارى در اين پارامتر بين ورزشكاران مبتدى در يِى برتر و غيربرتر ديده نشد. نتايج محاسبهشده مربوط به فشار وارد به قسمتهاى داخلى و خارجى با نشان داد كه ميانگينِ بيشترين فشار در قسمت داخلى بِاى تكيه گاه، هنگامى كه آزمودنىها با پِاى برتر اقدام به شوت روى پٍا كردند، 19/91 ياسكال است كه اين مقدار براى پِاى تكيه كاه غيربرتر

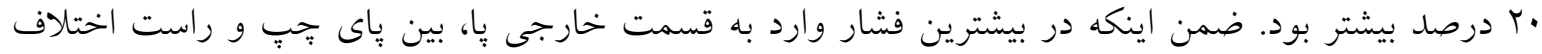

$$
\text { معنادارى مشاهده شد (جدول (1). }
$$


جدول ا.ميانكين و انحر اف معيار يارامترهاى بيومكانيكى در پياى تكيه كاه برتر و غير برتر (استاندارد شده براساس وزن)

\begin{tabular}{|c|c|c|c|}
\hline 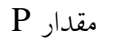 & شوت با پِاى غير برتر & شوت با ياى برتر & \\
\hline$* \cdot / \cdot 1 r$ & $\leftrightarrow \wedge / \mu \pm 1 \uparrow$ & $09 / 9 \pm 1 r / \Lambda$ & زاويه ساب تالار (deg) \\
\hline$* \cdot \cdots 1$ & $19 / \mu T \pm \Lambda / r$ & $\Delta / ৭ \pm r / r$ & زاويه محور پيا (deg) \\
\hline$\cdot /$ TSV & $\Lambda / \Delta \pm \Psi / \Delta$ & V/rTI & ضربه وارده به عقب پيا (N*s) \\
\hline$\cdot / M M_{Y}$ & $r / v \pm 1 / 4$ & $\varphi / \cdot 9 \pm 1 / \mu$ & ضربه وارده به وسط يا (N*s) \\
\hline$\cdot / 491$ & $\Lambda V / V \pm \Delta / 9$ & $\Lambda \Lambda / \Delta \pm \psi / \mu$ & ضربه وارده به جلوى يا (N*s) \\
\hline$* \cdot \cdots \Delta$ & $r Y / Q V \pm I Y / V$ & $r Y / r \pm \Lambda / r$ & حداكثر فشار وارد به خارج ڤا (Pa) \\
\hline$\cdot / 1$ & $r \mu / \wedge \pm q / r$ & $19 / \mathrm{V} \pm \mathrm{V} / 0$ & حداكثر فشار وارد به داخل پا (Pa) \\
\hline$* \cdot 1 \cdot 44$ & $T V / I Q$ & $M Y / M Y^{k}$ & زمان وقوع حداكثر نيرو در پياى تكيه كاه (.) \\
\hline
\end{tabular}

اطلاعات مربوط به زمانبندى مراحل مختلف قرارگرفتن ياى تكيه گاه روى زمين، در حين شوت روى يا با پِاى

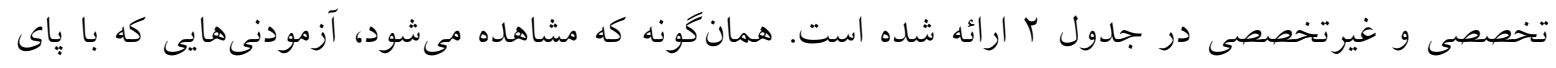
غيربرتر خود اقدام به زدن شوت مى كنند، براى كاملشدن حركت، زمان بيشترى را در فاز استقرار صرف مىكنند. ضمن اينكه، در لحظهُ جداشدن ياشنه از زمين، بين دو ياى تكيه كاه برتر و غيربرتر اختلاف معنى دار ديده مىشود.

جدول r ـ ميانگين زمان رسيدن پِاى تكيه كاه به مراحل مختلف فاز استقرار در حين شوت با باى برتر و غير برتر

\begin{tabular}{|c|c|c|c|}
\hline Pقدار P P P & ياى تكيه كاه غير برتر (ثانيه) & پياى تكيه گاه برتر (ثانيه) & مراحل فاز استقرار \\
\hline & - & - & لحظه اولين برخورد \\
\hline$\cdot / 0$ & $\cdot / \cdot \wedge \mathrm{V}$ & $\cdot / \cdot \mathrm{v} \wedge$ & لحظه اولين برخورد كف پيا \\
\hline$\cdot / \mathrm{V} \cdot$ & ( &.$/ M T$ & لحظه برخورد كامل كف يا \\
\hline$* . / \cdot r q$ & $\cdot 109$ & ש r & لحظه بلند شدن يّاشنه \\
\hline 1 & $1 / 90$ & $1 / T 0$ & لحظه جدا شدن يا \\
\hline
\end{tabular}

مقدار نيروى عمودى وارد بر باى تكيه گاه در مراحل مختلف فاز استقرار نيز در اين مطالعه محاسبه شد و در دو پاى برتر و غيربرتر تحت مقايسه قرارگرفت. ميانگين نيروى عمودى وارد به ياى تكيه كاه غيربرتر در ابتداى برخورد پا به صفحهُ فشار تا انتهاى لحظهُ بلندشدن پياشنه، بيشتر از ياى تكيه كاه برتر بود و در مقدار اين نيرو، در دامنهُ برخورد اوليه تا برخورد كف يا، اختلاف معنادارى بين يّاى برتر و غيربرتر مشاهده شد (شكل (1). 


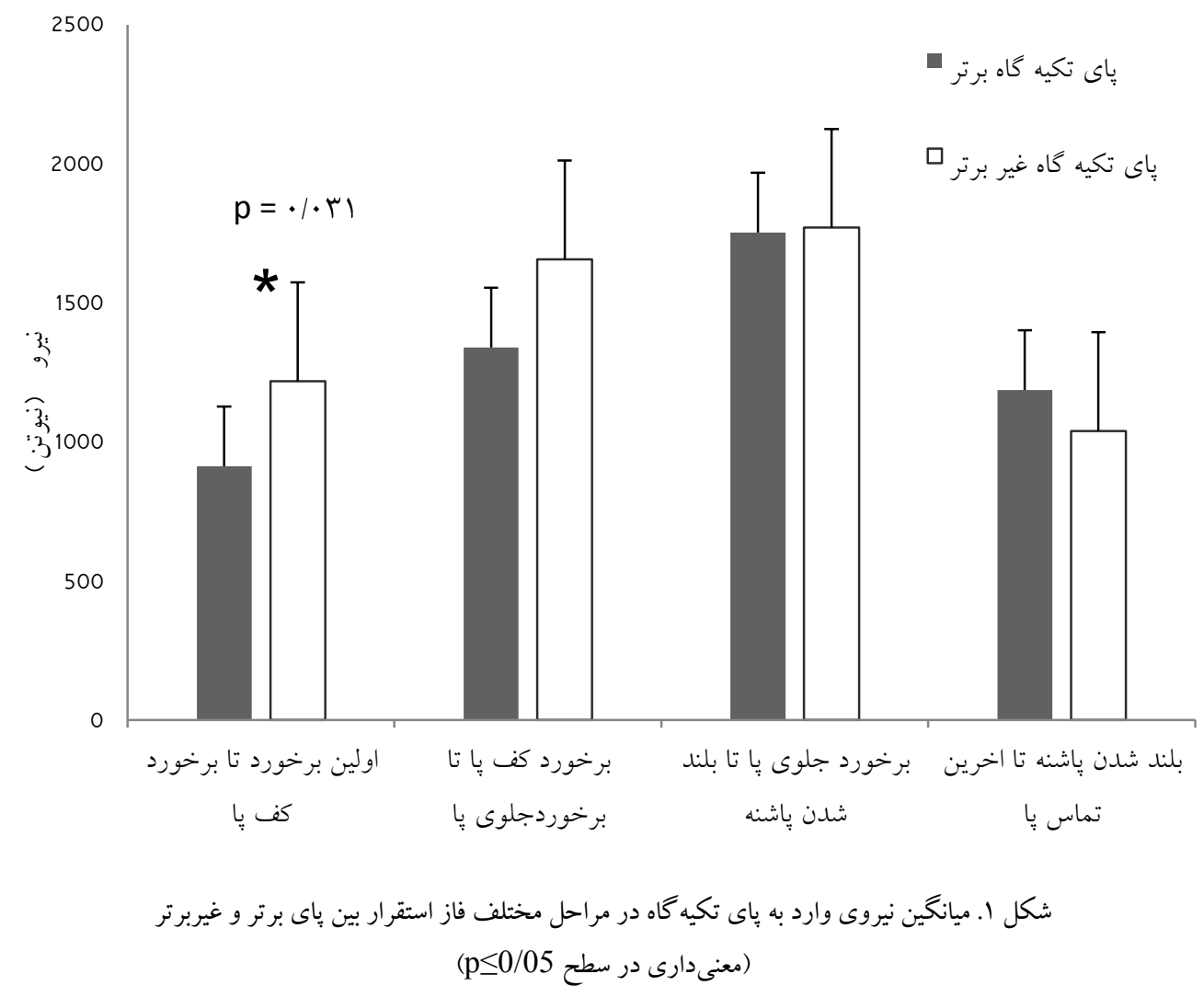

هدف اين مطالعه، مقايسة متتخبى از بارامترهاى بيومكانيكى و ميزان خطر آسيب مجّ باى تكيه كاهِ برتر و غيربرتر

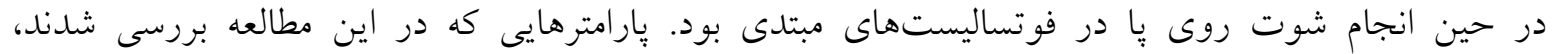

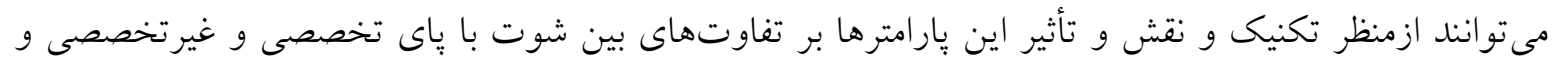

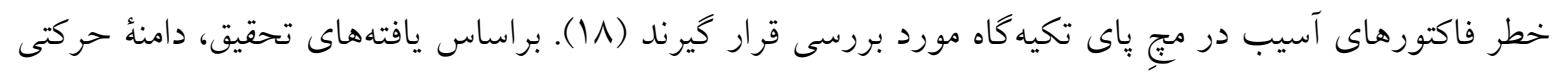

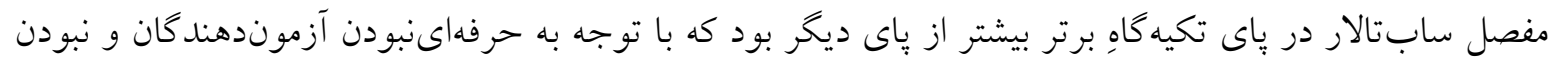

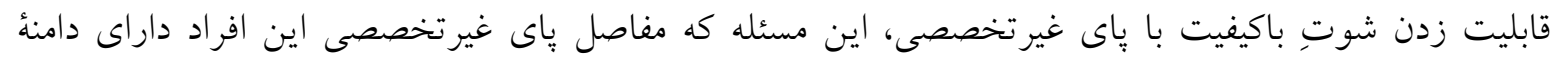

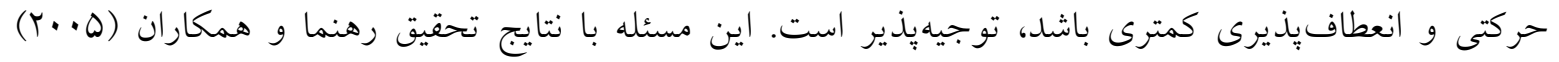

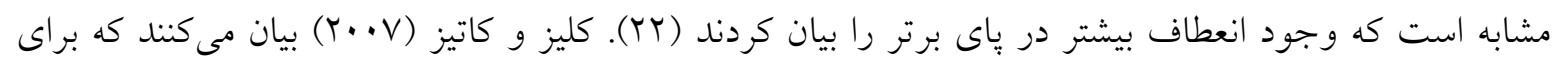

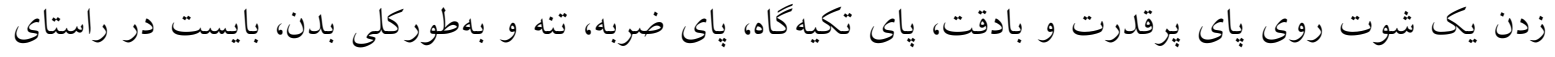

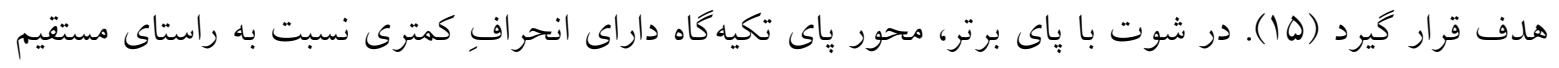

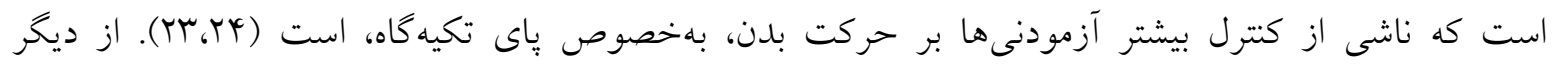


يارامترهاى تحت بررسى در اين يزوهش، مقدار ضربة وارد به قسمتهاى مختلف بإى تكيه كاه بود. زمانى كه

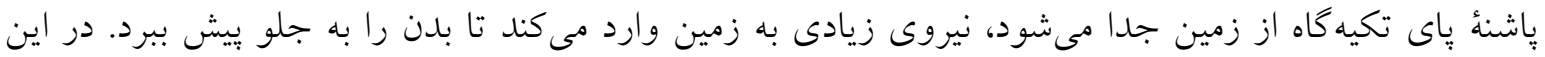
تحقيق نيز مشاهده شد كه مقدار ضربة وارد به قسمت فوقانى با اختلاف درخور توجهى با ديكر مناطق داشت.

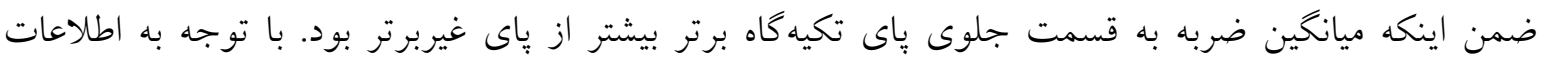

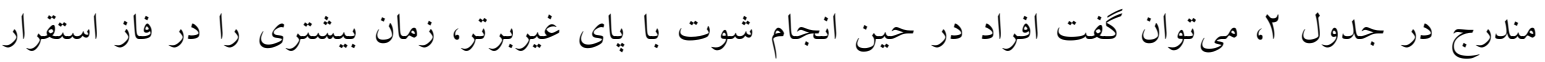

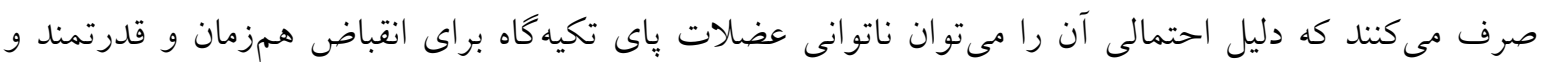

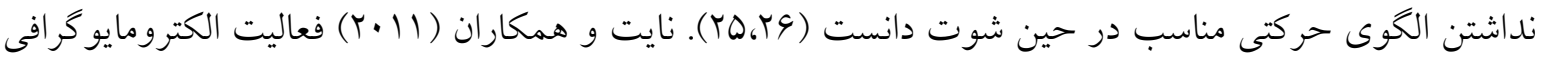

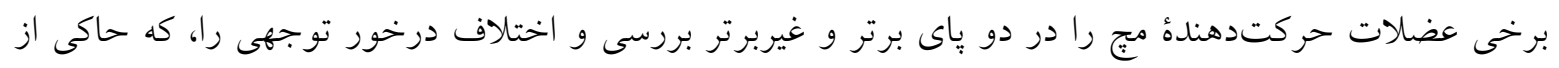
وجود قدرت بيشتر در عضلات ياى برتر بود، گزارش كردند (YV).

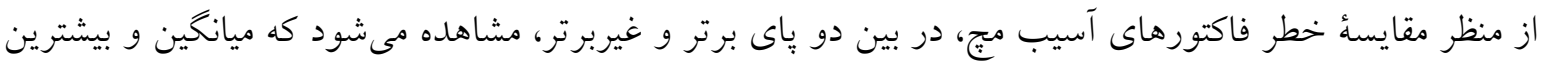

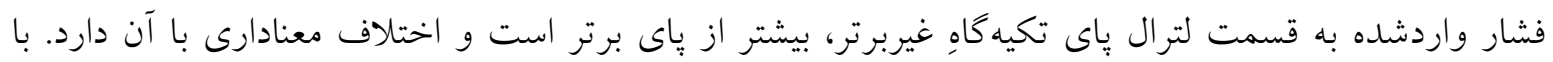

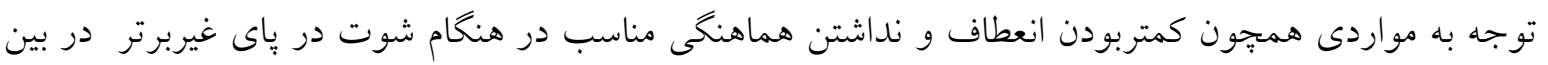

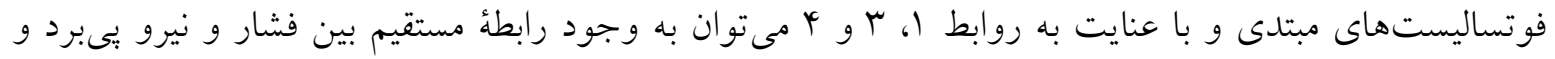

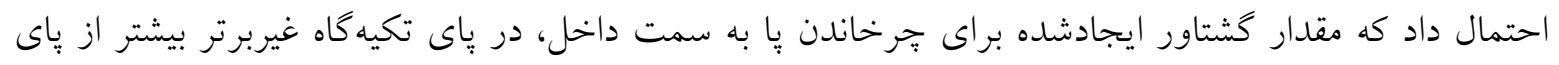

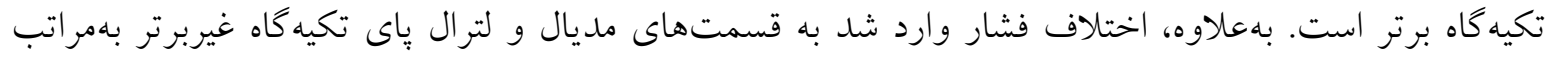

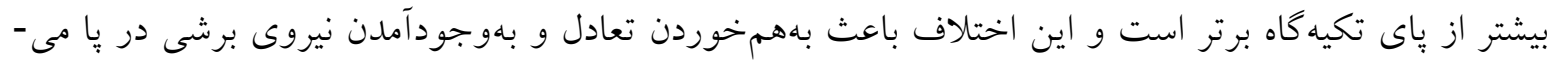

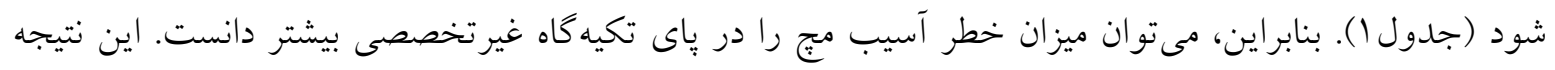

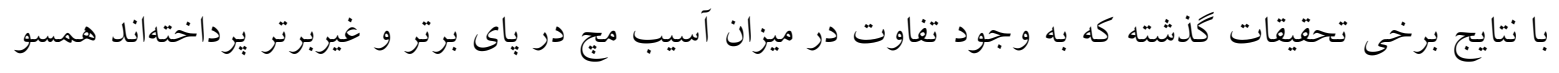

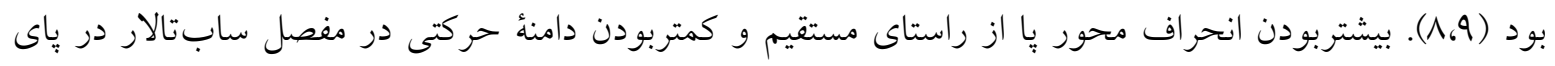

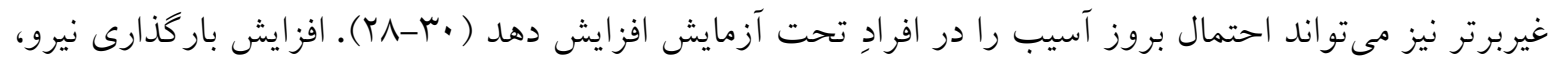

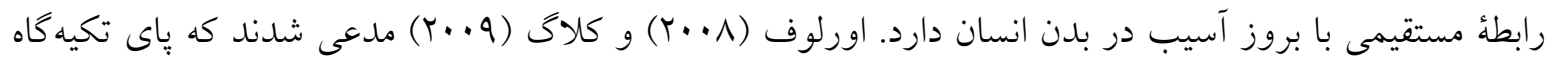

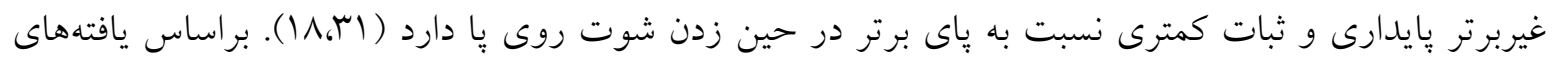

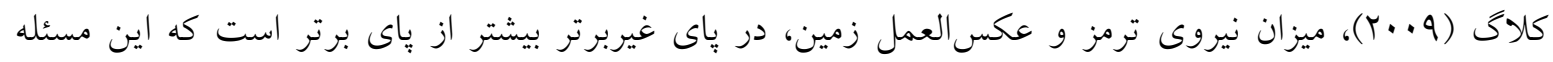

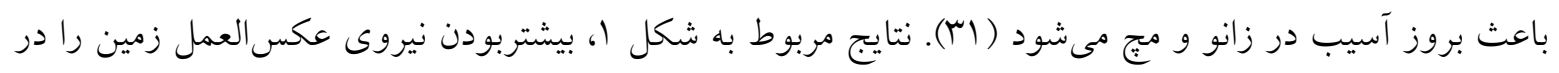

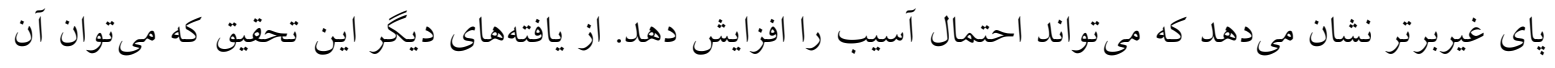

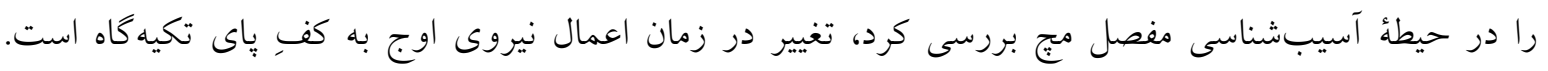

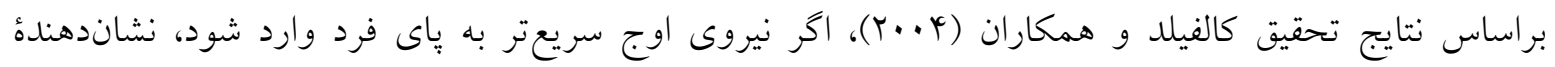

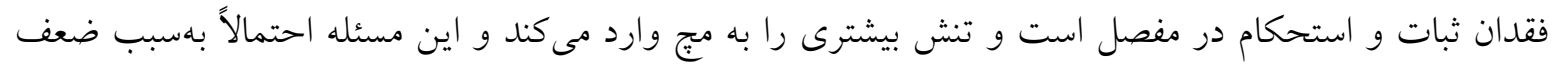




$$
\begin{aligned}
& \text { در كتترل حركت رخ مىدهد (با). با توجه به جدول ا، مىتوان مشاهده كرد كه بيشترين نيرو در ياى تكيه گاه } \\
& \text { غيربرتر زودتر از پایى برتر بروز بيدا كرده است و اين مىتواند در جريان مسابقه و در تكرارهاى مكرر، زمانى كه }
\end{aligned}
$$

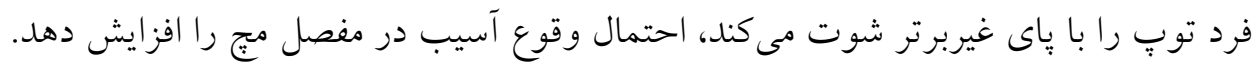

$$
\begin{aligned}
& \text { نتيجه كيرى } \\
& \text { با توجه به نتايج تحقيق، مىتوان كفت بين باى تكيه گاهِ برتر و غيربرتر در بين فوتساليستهاى مبتدى، تفاوتهاى }
\end{aligned}
$$

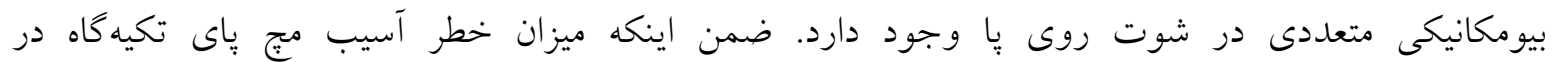

$$
\begin{aligned}
& \text { فوتساليستهاى مبتدى در زدن شوت روى يا با پاى غيربرتر بهمراتب بيشتر از پِاى برتر است. }
\end{aligned}
$$

1. Nielsen, A.B., Yde, J. (1989). Epidemiology and traumatology of injuries in soccer. The American Journal of Sports Medicine. 17(6):803-7.

2. Jørring, K. (1980). Osteoarthritis of the hip: epidemiology and clinical role. Acta Orthopaedica Scandinavica. 51(16):523-30.

3. Söderman, K., Alfredson, H., Pietilä, T., Werner, S. (2001). Risk factors for leg injuries in female soccer players: a prospective investigation during one out-door season. Knee Surgery, Sports Traumatology, Arthroscopy. 9(5):31321.

4. Ekstrand, J., Gillquist, J. (1983). The avoidability of soccer injuries. International Journal of Sports Medicine. 4(02):124-8.

5. Chomiak, J., Junge, A., Peterson, L., Dvorak, J. (2000). Severe injuries in football players . Influencing factors. The American Journal of Sports Medicine. 28(5):58-68.

6. Backous, D.D., Friedl, K.E., Smith, N.J., Parr, T.J., Carpine, W.D. (1988). Soccer injuries and their relation to physical maturity. American Journal of Diseases of Children. 142(8):839-42.

7. Östenberg, A., Roos, H. (2000). Injury risk factors in female European football. A prospective study of 123 players during one season. Scandinavian Journal of Medicine \& Science in Sports. 10(5):279-85.

8. Baumhauer, J.F., Alosa, D.M., Renström, P.A., Trevino, S., Beynnon, B. (1995). A prospective study of ankle injury risk factors. The American Journal of Sports Medicine. 23(5):564-70.

9. Ekstrand, J., Gillquist, J. (1982). Soccer injuries and their mechanisms: a prospective study. Medicine and Science in Sports and Exercise. 15(3):267-70.

10. Orchard, J.W. (2001). Intrinsic and extrinsic risk factors for muscle strains in australian football. The American Journal of Sports Medicine. 29(3):300-3.

11. Beynnon, B.D., Renström, P.A., Alosa, D.M., Baumhauer, J.F., Vacek, P.M. (2001). Ankle ligament injury risk factors: a prospective study of college athletes. Journal of Orthopaedic Research. 19(2):213-20.

12. Seil, R., Rupp, S., Tempelhof, S., Kohn, D. (1998). Sports injuries in team handball A one-year prospective study of sixteen men's senior teams of a superior nonprofessional level. The American Journal of Sports Medicine 26(5):681-7.

13. Surve, I., Schwellnus, M.P., Noakes, T., Lombard, C. (1994). A fivefold reduction in the incidence of recurrent ankle sprains in soccer players using the Sport-Stirrup orthosis. The American Journal of Sports Medicine. 22(5):601-6.

14. Barfield, W.R., Kirkendall, D.T., Yu, B. (2002). Kinematic instep kicking differences between elite female and male soccer players. Journal of Sports Science and Medicine. 1(3):72-9.

15. Kellis, E., Katis, A. (2007). Biomechanical characteristics and determinants of instep soccer kick. Journal of Sports Science and Medicine. 6(2):154-65.

16. Katis, A., Kellis, E. (2010). Three-dimensional kinematics and ground reaction forces during the instep and outstep soccer kicks in pubertal players. Journal of Sports Sciences. 28(11):1233-41.

17. Orloff, H., Sumida, B., Chow, J., Habibi, L., Fujino, A., Kramer, B. (2008). Ground reaction forces and kinematics of plant leg position during instep kicking in male and female collegiate soccer players. Sports Biomechanics. 7(2): 238-47.

18. Lees, A., Asai, T., Andersen, T.B., Nunome, H., Sterzing, T. (2010). The biomechanics of kicking in soccer: A review. Journal of Sports Sciences. 28(8):805-17.

19. Dörge, H.C., Andersen, T.B., SØrensen, H., Simonsen, E.B. (2002). Biomechanical differences in soccer kicking with the preferred and the non-preferred leg. Journal of Sports Sciences. 20(4):293-99.

20. Murphy, D.F., Connolly, D.A. J., Beynnon, B.D. (2003). Risk factors for lower extremity injury: a review of the literature. British Journal of Sports Medicine. 37(1):13-29.

21. Ekstrand, J. (2008). Epidemiology of football injuries. Science \& Sports. 23(2):73-77.

22. Rahnama, N., Lees, A., Bambaecichi, E. (2005). A comparison of muscle strength and flexibility between the preferred and non-preferred leg in English soccer players. Ergonomics. 48(11-14):1568-75. 
23. Nunome, H., Lake, M., Georgakis, A., Stergioulas, L.K. (2006). Impact phase kinematics of instep kicking in soccer. Journal of Sports Sciences. 24(1):11-22.

24. Davids, K., Lees, A., Burwitz, L. (2000). Understanding and measuring coordination and control in kicking skills in soccer: Implications for talent identification and skill acquisition. Journal of Sports Sciences. 18(9):703-14.

25. Masuda, K., Kikuhara, N., Takahashi, H., Yamanaka, K. (2003). The relationship between muscle cross-sectional area and strength in various isokinetic movements among soccer players. Journal of Sports Sciences. 21(10):85158.

26. Maraj, B.K., Morrison, Z.J., JiaYi, C., Davids, K. (2006). The perceptual-motor regulation of kicking in soccer. International Journal of Sport Psychology. 37(2/3):157-85.

27. Knight, A.C., Weimar, W.H. (2011). Difference in response latency of the peroneus longus between the dominant and nondominant legs. Journal of Sport Rehabilitation. 20(3):321-32.

28. Kvist, M. (1994). Achilles tendon injuries in athletes. Sports Medicine. 18(3):173-201.

29. Siegler, S., Chen, J., Schneck, C.D. (1988). The three-dimensional kinematics and flexibility characteristics of the human ankle and subtalar joints—Part I: Kinematics. Journal of Biomechanical Engineering. 110(4):364-73.

30. Krivickas, L.S. (1997). Anatomical factors associated with overuse sports injuries. Sports Medicine. 24(2):132-46.

31. Clagg, S.E., Warnock, A., Thomas, J.S. (2009). Kinetic analyses of maximal effort soccer kicks in female collegiate athletes. Sports Biomechanics. 8(2):141-53.

32. Caulfield, B., Garrett, M. (2004). Changes in ground reaction force during jump landing in subjects with functional instability of the ankle joint. Clinical Biomechanics. 19(6):617-21. 